\title{
Dispersing cocycles and mixing flows under functions
}

\author{
by
}

\author{
Klaus Schmidt (Wien)
}

\begin{abstract}
Let $T$ be a measure-preserving and mixing action of a countable abelian group $G$ on a probability space $(X, \mathcal{S}, \mu)$ and $A$ a locally compact second countable abelian group. A cocycle $c$ : $G \times X \rightarrow A$ for $T$ disperses if $\lim _{g \rightarrow \infty} c(g, \cdot)-\alpha(g)=\infty$ in measure for every map $\alpha: G \rightarrow A$. We prove that such a cocycle $c$ does not disperse if and only if there exists a compact subgroup $A_{0} \subset A$ such that the composition $\theta \circ c: G \times X \rightarrow A / A_{0}$ of $c$ with the quotient map $\theta: A \rightarrow A / A_{0}$ is trivial (i.e. cohomologous to a homomorphism $\left.\eta: G \rightarrow A / A_{0}\right)$.

This result extends a number of earlier characterizations of coboundaries and trivial cocycles by tightness conditions on the distributions of the maps $\{c(g, \cdot): g \in G\}$ and has implications for flows under functions: let $T$ be a measure-preserving ergodic automorphism of a probability space $(X, \mathcal{S}, \mu), f: X \rightarrow \mathbb{R}$ be a nonnegative Borel map with $\int f d \mu=1$, and $T^{f}$ be the flow under the function $f$ with base $T$. Our main result implies that, if $T$ is mixing and $T^{f}$ is weakly mixing, or if $T$ is ergodic and $T^{f}$ is mixing, then the cocycle $\mathbf{f}: \mathbb{Z} \times X \rightarrow \mathbb{R}$ defined by $f$ disperses. The latter statement answers a question raised by Mariusz Lemańczyk in [7].
\end{abstract}

\section{Dispersion of cocycles}

DeFinition 1.1. Let $T: g \mapsto T_{g}$ be a measure-preserving action of a countable additive abelian group $G$ on a standard probability space $(X, \mathcal{S}, \mu)$, and let $A$ be a locally compact second countable additive abelian group with identity element 0 . A Borel map $c: G \times X \rightarrow A$ is a cocycle for $T$ if

$$
c\left(g, T_{h} x\right)+c(h, x)=c(g+h, x)
$$

for every $g, h \in G$ and $x \in X$. Two cocycles $c, c^{\prime}: G \times X \rightarrow A$ are cohomologous if there exists a Borel map $b: X \rightarrow A$ such that

$$
c(g, x)=c^{\prime}(g, x)+b\left(T_{g} x\right)-b(x)
$$

2000 Mathematics Subject Classification: 37A05, 37A10, 37A20, 37A25, 37H05.

Key words and phrases: boundedness, tightness and dispersion of cocycles, mixing properties of flows.

I would like to thank the Department of Mathematics, University of Torun, for hospitality while this work was done, and to Mariusz Lemańczyk (who pointed out this problem to me) and Jon Aaronson for stimulating discussions. 
for every $g \in G$ and $\mu$-a.e. $x \in X$. The map $b$ in (1.1) is called a transfer function. If $c$ is cohomologous to the zero cocycle $c^{\prime} \equiv 0$ then $c$ is a coboundary with transfer (or cobounding) function $b$.

Let $c: G \times X \rightarrow A$ be a cocycle. The cocycle $c$ is a homomorphism if the map $c(g, \cdot): X \rightarrow A$ is constant for every $g \in G$, and $c$ is trivial if it is cohomologous to a homomorphism.

The cocycle $c$ is bounded (in measure) on a subset $H \subset G$ if there exists, for every $\varepsilon>0$, a compact subset $C \subset A$ with

$$
\mu(\{x: c(g, x) \in C\})>1-\varepsilon
$$

for every $g \in H$.

The cocycle $c$ is translation-bounded on a subset $H \subset G$ if there exist, for every $\varepsilon>0$, a map $\alpha: H \rightarrow A$ and a compact subset $C \subset A$ with

$$
\mu(\{x: c(g, x)-\alpha(g) \in C\})>1-\varepsilon
$$

for every $g \in H$.

If $H=G$ in (1.2) or (1.3) then $c$ is said to be bounded or translationbounded, respectively.

Finally, the cocycle $c$ disperses if $\lim _{g \rightarrow \infty} c(g, \cdot)-\alpha(g)=\infty$ in measure for every map $\alpha: G \rightarrow A$ or, equivalently, if

$$
\lim _{g \rightarrow \infty} \sup _{a \in A} \mu(\{x \in X: c(g, x)-a \in C\})=0
$$

for every compact set $C \subset A$.

It has long been known that a cocycle $c: G \times X \rightarrow \mathbb{R}$ is a coboundary if and only if it is bounded in the sense of Definition 1.1 (cf. [9, Theorem 11.8]). More generally, if $A$ is a locally compact second countable abelian group and $c: G \times X \rightarrow A$ a bounded cocycle, then $c$ is cohomologous to a cocycle taking values in a compact subgroup $A_{0} \subset A$ (for extensions of this result with varying degrees of generality see [8, Theorem 5.2], [10, Theorem 4.7] and [1]). Furthermore, if $A=\mathbb{R}$, then $c$ is trivial if and only if it is translation-bounded (cf. [8, Theorem 6.2]).

More recently it was shown that, if $T$ is mixing, then boundedness (or translation-boundedness) of a cocycle $c: G \times X \rightarrow \mathbb{R}$ on an infinite subset $H \subset G$ also implies triviality. The first published result in this direction is $[1$, Theorem 2], where it is proved that, for a mixing action of $G=\mathbb{Z}$, translation-boundedness of a cocycle $c: \mathbb{Z} \times X \rightarrow \mathbb{R}$ on some infinite subset $H \subset G$ implies triviality of $c$, and boundedness of $c$ on $H$ implies that $c$ is a coboundary. These results can break down for $\mathbb{Z}$-actions which are only mildly mixing (cf. [1]).

In this note we prove the following extension of [1, Theorem 2].

TheOREM 1.2. Let $T$ be a measure-preserving and mixing action of a countable abelian group $G$ on a standard probability space $(X, \mathcal{S}, \mu), A$ a 
locally compact second countable abelian group and $c: G \times X \rightarrow A$ a cocycle for $T$. The following conditions are equivalent:

(1) The cocycle $c$ does not disperse (cf. (1.4)).

(2) There exists a compact subgroup $A_{0} \subset A$ such that the composition $\theta \circ c: G \times X \rightarrow A / A_{0}$ of $c$ with the quotient map $\theta: A \rightarrow A / A_{0}$ is a trivial cocycle.

For the proof of Theorem 1.2 we need a little bit of notation. Let $T$ be a continuous action of a countable abelian group $G$ on a compact metrizable space $X$ and $\mu$ be a $T$-invariant Borel probability measure on $X$. We denote by $\Delta=\{(x, x): x \in X\}$ the diagonal in $X \times X$ and define the "diagonal" probability measure $\mu_{\Delta}$ on $X \times X$ by setting

$$
\mu_{\Delta}(\{(x, x): x \in B\})=\mu(B)
$$

for every Borel set $B \subset X$. For every $g \in G$, the "off-diagonal" probability measure

$$
\nu_{g}=\left(T_{g} \times \operatorname{Id}_{X}\right)_{*}\left(\mu_{\Delta}\right)
$$

is the self-joining of $\mu$ supported on the graph $\left\{\left(T_{g} x, x\right): x \in X\right\}$ of $T_{g}$.

Theorem 1.2 is an easy consequence of the following proposition.

Proposition 1.3. Let $T$ be a continuous action of a countable abelian group $G$ on a compact metrizable space $X, \mu$ a T-invariant and weakly mixing Borel probability measure on $X, A$ a locally compact second countable abelian group and $c: G \times X \rightarrow A$ a cocycle for $T$. Suppose that there exists a sequence $\left(h_{n}: n \geq 1\right)$ in $G$ with the following properties:

(1) $\lim _{n \rightarrow \infty} \nu_{h_{n}}=\mu \times \mu$ in the topology of weak convergence;

(2) There exist an $\varepsilon>0$, a compact set $C \subset A$ and elements $\alpha_{n} \in A$, $n \geq 1$, with

$$
\mu\left(\left\{x \in X: c\left(h_{n}, x\right)-\alpha_{n} \in C\right\}\right) \geq \varepsilon \quad \text { for every } n \geq 1 .
$$

Then there exists a compact subgroup $A_{0} \subset A$ such that the composition $\theta \circ c: G \times X \rightarrow A / A_{0}$ of $c$ with the quotient map $\theta: A \rightarrow A / A_{0}$ is trivial.

For the proof of Proposition 1.3 we require an elementary lemma closely related to [10, Lemma 4.4].

LEMMA 1.4. Let $T$ be a measure-preserving and ergodic action of a countable abelian group $G$ on a standard probability space $(X, \mathcal{S}, \mu), A$ a locally compact second countable abelian group and $c: G \times X \rightarrow A$ a cocycle for $T$. We define the skew-product action $T^{(c)}$ of $G$ on $Y=X \times A$ by setting

$$
T_{g}^{(c)}(x, a)=\left(T_{g} x, c(g, x)+a\right)
$$

for every $g \in G$ and $(x, a) \in Y$. 
If there exists a $T^{(c)}$-invariant probability measure $\varrho$ on $Y$ with $\pi_{*}(\varrho)=\mu$ (where $\pi: Y \rightarrow X$ is the first coordinate projection), then $c$ is cohomologous to a cocycle $c^{\prime}$ taking values in some compact subgroup $A_{0} \subset A$.

Conversely, if $c$ is cohomologous, with transfer function $b$, to a cocycle $c^{\prime}: G \times X \rightarrow A_{0}$, where $A_{0} \subset A$ is a compact subgroup with normalized Haar measure $\lambda_{A_{0}}$, then the probability measure $\varrho$ on $Y$, defined by

$$
\int f d \varrho=\iint f(x, a+b(x)) d \lambda_{A_{0}}(a) d \mu(x)
$$

for every bounded Borel map $f: Y \rightarrow \mathbb{R}$, is $T^{(c)}$-invariant and $\pi_{*}(\varrho)=\mu$.

Proof. Choose a Borel measurable family of probability measures $\left\{\varrho_{x}\right.$ : $x \in X\}$ on $A$ such that

$$
\int f d \varrho=\iint f(x, t) d \varrho_{x}(t) d \varrho(x)
$$

for every bounded Borel map $f: Y \rightarrow A$. Since $\varrho$ is $T^{(c)}$-invariant,

$$
\varrho_{T_{g} x}(B+c(g, x))=\varrho_{x}(B) \quad \text { for } \mu \text {-a.e. } x \in X,
$$

for every Borel set $B \subset A$ and every $g \in G$. We fix a nonnegative continuous map $\phi: A \rightarrow \mathbb{R}$ with compact support such that $\phi(0)>0$. For every $x \in X$, the map $a \mapsto \int \phi(a+s) d \varrho_{x}(s)=\psi(x, a)$ from $A$ to $\mathbb{R}$ is continuous, not everywhere equal to zero, and vanishes at infinity. Furthermore, the resulting Borel map $\psi: X \times A \rightarrow \mathbb{R}$ is $T^{(c)}$-invariant, and for some $\varepsilon>0$ the Borel set

$$
K=\{(x, a) \in Y: \psi(x, a) \geq \varepsilon\}
$$

is nonempty and again $T^{(c)}$-invariant. For every $x \in X$, the set

$$
K_{x}=\{a \in A:(x, a) \in K\}
$$

and the subgroup

$$
A_{x}=\left\{a \in A: a+K_{x}=K_{x}\right\}
$$

are both compact, and the ergodicity of $T$ and the $T^{(c)}$-invariance of $K$ imply that there exists a compact subgroup $A_{0} \subset A$ with $A_{x}=A_{0}$ for $\mu$-a.e. $x \in X$. By using one of the standard selection theorems (cf. e.g. Kunugui's theorem in [5]-[6]) we can choose a Borel map $b: X \rightarrow A$ with $b(x) \in K_{x}$ for $\mu$-a.e. $x \in X$ and conclude that $c(g, x)+b(x)-b\left(T_{g} x\right) \in A_{0}$ for every $g \in G$ and $\mu$-a.e. $x \in X$.

The final statement of the lemma is obvious.

Proof of Proposition 1.3. Let $\bar{T}: g \mapsto T_{g} \times T_{g}$ denote the diagonal action of $G$ on $\bar{X}=X \times X$, and let $\bar{c}: G \times \bar{X} \rightarrow A$ be the cocycle

$$
\bar{c}\left(g,\left(x_{1}, x_{2}\right)\right)=c\left(g, x_{1}\right)-c\left(g, x_{2}\right)
$$

for $\bar{T}$. The cocycle equation (2.1) yields

$$
c\left(g, T_{h_{n}} x\right)-c(g, x)=c\left(h_{n}, T_{g} x\right)-c\left(h_{n}, x\right)
$$


for every $g \in G$ and $n \geq 1$. For every $n \geq 1$ we define $b_{n}: \bar{X} \rightarrow A$ by $b_{n}\left(x_{1}, x_{2}\right)=c\left(h_{n}, x_{2}\right)-\alpha_{n}$ and conclude from (1.7) that

$$
\bar{c}\left(g,\left(x_{1}, x_{2}\right)\right)=b_{n} \circ \bar{T}_{g}\left(x_{1}, x_{2}\right)-b_{n}\left(x_{1}, x_{2}\right)
$$

for every $g \in G$ and $\left(x_{1}, x_{2}\right) \in\left(T_{h_{n}} \times \operatorname{Id}_{X}\right)(\Delta)$, i.e. $\bar{c}$ is a coboundary with cobounding function $b_{n}$ with respect to the $\bar{T}$-invariant measure $\nu_{n}=\nu_{h_{n}}$. We denote by $\varrho_{n}$ the probability measure on $Y=\bar{X} \times A$ with

$$
\varrho_{n}\left(\left\{\left(T_{h_{n}} x, x, b_{n}\left(T_{h_{n}} x, x\right)\right): x \in B\right\}\right)=\mu(B)
$$

for every Borel set $B \subset X$ and observe as in Lemma 1.4 that $\varrho_{n}$ is the unique $T^{(c)}$-invariant probability measure supported on the graph of $b_{n}$ with $\pi_{*}\left(\varrho_{n}\right)=\nu_{n}$, where $\pi: \bar{X} \times A \rightarrow \bar{X}$ is the projection map. If

$$
A_{n}=\left\{x \in X: b_{n}(x) \in C\right\},
$$

where $C \subset A$ is the compact set appearing in (1.6), then

$$
\varrho_{n}(\bar{X} \times C)=\nu_{n}\left(T_{h}^{-1} A_{n} \times A_{n}\right)=\mu\left(A_{n}\right) \geq \varepsilon
$$

for every $n \geq 1$.

By going over to a subsequence of $\left(h_{n}\right)$, if necessary, we may take it that the sequence of probability measures $\left(\varrho_{n}\right)$ converges vaguely to a finite measure $\varrho$ on $Y$, i.e. $\lim _{n \rightarrow \infty} \int f d \varrho_{n}=\int f d \varrho$ for every continuous function $f: Y \rightarrow A$ with compact support. According to (1.8),

$$
\varrho(\bar{X} \times C) \geq \limsup _{n \rightarrow \infty} \varrho_{n}(\bar{X} \times C) \geq \varepsilon,
$$

which implies that $\varrho$ is nonzero. We set $\nu=\pi_{*}(\varrho)$ and claim that

$$
\nu(B) \leq(\mu \times \mu)(B)
$$

for every Borel set $B \subset \bar{X}$.

Indeed, let $f: \bar{X} \rightarrow A$ be a continuous function, $\mathcal{U} \subset A$ an open neighbourhood of the identity with compact closure, $\left(D_{m}: m \geq 1\right)$ a sequence of compact subsets of $A$ with $D_{m+1} \supset D_{m}+\mathcal{U}$ for every $m \geq 1$ and $\bigcup_{m \geq 1} D_{m}=A$, and let, for every $m \geq 1, \phi_{m}: A \rightarrow A$ be a continuous map with $\phi_{m}(a)=1$ for $a \in D_{m}$ and $\phi_{m}(a)=0$ for $a \notin D_{m}+\mathcal{U}$. We set $f_{m}\left(x_{1}, x_{2}, a\right)=f\left(x_{1}, x_{2}\right) \phi_{m}(a)$ and observe that

$$
\int f_{m} d \varrho=\lim _{n \rightarrow \infty} \int f_{m} d \varrho_{n} \leq \lim _{n \rightarrow \infty} \int f d \nu_{n}=\int f d(\mu \times \mu) .
$$

By letting $m \rightarrow \infty$ we obtain

$$
\int f d \nu=\sup _{m \geq 1} \int f_{m} d \varrho \leq \int f d(\mu \times \mu) .
$$

As $f$ was arbitrary, this proves (1.9).

Since each of the probability measures $\varrho_{n}$ is invariant under the skewproduct action

$$
\bar{T}_{g}^{(\bar{c})}\left(x_{1}, x_{2}, a\right)=\left(T_{g} x_{1}, T_{g} x_{2}, \bar{c}\left(g,\left(x_{1}, x_{2}\right)\right)+a\right)
$$


of $G$ on $Y$, the same $\left(^{1}\right)$ is true for $\varrho$, and hence the measure $\nu$ on $\bar{X}$ is invariant under $\bar{T}$. From (1.9) and the ergodicity of $\mu \times \mu$ it is clear that $(1 / \nu(\bar{X})) \nu=\nu^{\prime}$ is a $\bar{T}$-invariant probability measure on $\bar{X}$ which is absolutely continuous with respect to, and hence equal to, $\mu \times \mu$, and that the probability measure $\varrho^{\prime}=(1 / \varrho(Y)) \varrho=(1 / \nu(\bar{X})) \varrho$ on $Y$ is invariant under $\bar{T}^{(\bar{c})}$ and satisfies $\pi_{*}\left(\varrho^{\prime}\right)=\mu \times \mu$.

By Lemma 1.4 there exists a compact subgroup $A_{0} \subset A$ such that $\theta \circ \bar{c}$ is a coboundary, where $\theta: A \rightarrow A^{\prime}=A / A_{0}$ is the quotient map.

In order to simplify notation a little we set $\bar{c}^{\prime}=\theta \circ \bar{c}: G \times \bar{X} \rightarrow A^{\prime}$ and $c^{\prime}=\theta \circ c: G \times X \rightarrow A^{\prime}$. In the notation of $[8,(6.1)$ and Theorem $6.2(4)]$ we have proved that $j_{*}\left(c^{\prime}\right)=0$, i.e. $c^{\prime} \in \Gamma_{2}\left(A^{\prime}\right)$ in the notation of $[8$, (7.5)]. As $\mu$ is weakly mixing, the triple diagonal action $T \times T \times T$ of $G$ on $(X \times X \times X, \mu \times \mu \times \mu)$ is ergodic, and [8, Corollary 7.2] shows that $\Gamma_{2}\left(A^{\prime}\right)=$ $\Gamma_{0}\left(A^{\prime}\right)$ in the notation of $[8,(7.3)-(7.5)]$. Hence $c^{\prime}$ is a homomorphism in the terminology of [8] or is trivial in our terminology.

Proof of Theorem 1.2. We assume without loss in generality that $X$ is a compact metric space and that the $G$-action $T$ on $X$ is continuous (cf. [11]).

If (1.4) is violated, then there exist an $\varepsilon>0$, a compact set $C \subset A$, an infinite subset $H \subset G$, and elements $\alpha_{h} \in A, h \in H$, with

$$
\mu\left(\left\{x \in X: c(h, x)-\alpha_{h} \in C\right\}\right) \geq \varepsilon
$$

for every $h \in H$. We can thus choose a sequence $\left(h_{n}\right)$ in $H$ with $\lim _{n \rightarrow \infty} h_{n}$ $=\infty$ which satisfies the conditions of Proposition 1.3. Hence there exists a compact subgroup $A_{0} \subset A$ such that the composition $\theta \circ c: G \times X \rightarrow A / A_{0}$ of $c$ with the quotient map $\theta: A \rightarrow A / A_{0}$ is trivial. This proves that (1) $\Rightarrow(2)$, and the reverse implication $(2) \Rightarrow(1)$ is obvious.

2. Mixing flows under functions. In order to apply Theorem 1.2 (or, more precisely, Proposition 1.3) to mixing properties of flows under functions we let $T$ be a measure-preserving automorphism of a standard probability space $(X, \mathcal{S}, \mu)$ and $f: X \rightarrow \mathbb{R}$ a Borel map with $\int f d \mu=1$ and $f(x)>0$ for every $x \in X$. For every $n \in \mathbb{Z}$ and $x \in X$ we set

$$
\mathbf{f}(n, x)= \begin{cases}\sum_{k=0}^{n-1} f\left(T^{k} x\right) & \text { if } n>0 \\ 0 & \text { if } n=0 \\ -\mathbf{f}\left(-n, T^{n} x\right) & \text { if } n<0\end{cases}
$$

$\left({ }^{1}\right)$ In order to see this, consider the ring $\mathcal{R}$ of all Borel sets $B \subset Y$ with the property that $\bar{T}_{g}^{(\bar{c})}(B)$ has compact closure and boundary measure $\varrho\left(\partial\left(\bar{T}_{g}^{(\bar{c})} B\right)\right)=0$ for every $g \in G$. By assumption, $\varrho(B)=\lim _{n \rightarrow \infty} \varrho_{n}(B)=\lim _{n \rightarrow \infty} \varrho_{n}\left(\bar{T}_{g}^{(\bar{c})} B\right)=\varrho\left(\bar{T}_{g}^{(\bar{c})} B\right)$ for every $g \in G$ and $B \in \mathcal{R}$. Since $\mathcal{R}$ generates the Borel field of $Y$ this proves that $\varrho$ is $\bar{T}^{(\bar{c})}$-invariant. 
The resulting map $\mathbf{f}: \mathbb{Z} \times X \rightarrow \mathbb{R}$ is a cocycle for $T$ (or for the $\mathbb{Z}$-action $n \mapsto T^{n}$ on $\left.(X, \mathcal{S}, \mu)\right)$. We define an equivalence relation $R^{f}$ on $X \times \mathbb{R}$ by saying that

$$
(x, t) \sim\left(T^{n} x, t-\mathbf{f}(n, x)\right)
$$

for every $(x, t) \in X \times \mathbb{T}$ and $n \in \mathbb{Z}$. The "vertical" flow $S_{t}:\left(x, t^{\prime}\right) \mapsto\left(x, t+t^{\prime}\right)$, $\left(x, t^{\prime}\right) \in X \times \mathbb{R}, t \in \mathbb{R}$, preserves this equivalence relation and thus induces a flow $t \mapsto S_{t}^{f}$ on the space $(X \times \mathbb{R})_{R^{f}}$ of equivalence classes of $R^{f}$. The set

$$
X^{f}=\{(x, t): x \in X, 0 \leq t<f(x)\}
$$

intersects each equivalence class of $R^{f}$ in exactly one point and may thus be identified with $(X \times \mathbb{R})_{R^{f}}$. We denote by $\mathcal{S}^{f}$ the Borel field of $X^{f} \subset X \times \mathbb{R}$, write $\lambda$ for the Lebesgue measure on $\mathbb{R}, \mu^{f}$ for the restriction to $X^{f} \subset X \times \mathbb{R}$ of the product measure $\mu \times \lambda$, and $T^{f}$ for the measure-preserving flow induced by $S^{f}$ on the probability space $\left(X^{f}, \mathcal{S}^{f}, \mu^{f}\right)$. This flow is usually called the flow under the function $f$ with base $T$.

Several authors have studied conditions on $f$ for a given ergodic base $T$ which determine whether the flow $T^{f}$ is mixing (cf. e.g. [3], [4], [2], [7] and the references listed there). In [7] the author proves the following result under the additional hypothesis that $T$ is an irrational rotation on $X=\mathbb{R} / \mathbb{Z}$, and asks whether Corollary 2.1 (under hypothesis (2)) holds for more general classes of ergodic automorphisms ([7, Remarque 2]).

COROLlary 2.1. Let $T$ be a measure-preserving automorphism of a standard probability space $(X, \mathcal{S}, \mu), f: X \rightarrow \mathbb{R}$ be a Borel map with $\int f d \mu=1$ and $f(x)>0$ for every $x \in X$, and $T^{f}$ be the flow under $f$ with base $T$ on the probability space $\left(X^{f}, \mathcal{S}^{f}, \mu^{f}\right)$. Suppose that either of the following conditions is satisfied:

(1) $T$ is mixing and $T^{f}$ is weakly mixing;

(2) $T^{f}$ is mixing.

Then the cocycle $\mathbf{f}: \mathbb{Z} \times X \rightarrow \mathbb{R}$ in (2.1) disperses in the sense of (1.4).

Proof. If $T$ is mixing, then Theorem 1.2 with $G=\mathbb{Z}$ and $A=\mathbb{R}$ shows that the cocycle $\mathbf{f}$ either disperses or is trivial, in which case the flow $T^{f}$ is not weakly mixing.

In order to prove dispersion of $\mathbf{f}$ under hypothesis (2) we may replace $f$ by a cohomologous function $f^{\prime}=f+b \circ T-b$ such that $b: X \rightarrow \mathbb{R}$ is measurable and $f^{\prime}$ is bounded above and below by positive constants; this will affect neither the hypotheses nor the conclusions of the corollary. We assume therefore without loss of generality that there exist positive constants $c_{1}<c_{2}$ such that $c_{1} \leq f(x) \leq c_{2}$ for every $x \in X$. 
Define a map $F: X^{f} \rightarrow \mathbb{R}$ by

$$
F(x, s)=1 / f(x)
$$

for every $x \in X$ and $s \in[0, f(x))$ and consider the cocycle $c: \mathbb{R} \times X^{f} \rightarrow \mathbb{R}$ given by

$$
c(t, z)= \begin{cases}\int_{0}^{t} F\left(T_{s}^{f} z\right) d s & \text { if } t>0 \\ 0 & \text { if } t=0 \\ -c\left(-t, T_{t}^{f} z\right) & \text { if } t<0\end{cases}
$$

For every positive $t$ we denote by $d(t, z)$ the number of intersections of the set $\{(x, 0): x \in X\} \subset X^{f}$ with the trajectory $\left\{T_{s}^{f} z: 0 \leq s<t\right\}$. Then

$$
|c(t, z)-d(t, z)| \leq 2 \quad \text { and } \quad|t-\mathbf{f}(d(t,(x, s)), x)| \leq 2 c_{2}
$$

for every $t \geq 0$ and $z=(x, s) \in X^{f}$, and

$$
\int c(t, z) d \mu^{f}(z)=t
$$

for every $t \in \mathbb{R}$.

Suppose that (1.4) is not satisfied, i.e. that there exist an $\varepsilon>0$, a constant $L>0$, an increasing sequence $\left(m_{n}\right)$ of positive integers, and a sequence $\left(t_{n}\right)$ in $\mathbb{R}$ with

$$
\mu\left(\left\{x \in X:\left|\mathbf{f}\left(m_{n}, x\right)-t_{n}\right| \leq L\right\}\right) \geq \varepsilon
$$

for every $n \geq 1$. According to (2.3) and (2.5),

$$
\mu^{f}\left(\left\{(x, s) \in X^{f}:\left|\mathbf{f}\left(d\left(t_{n},(x, s)\right), x\right)-\mathbf{f}\left(m_{n}, x\right)\right| \leq L+2 c_{2}\right\}\right) \geq c_{1} \varepsilon,
$$

hence

$$
\mu^{f}\left(\left\{z \in X^{f}:\left|d\left(t_{n}, z\right)-m_{n}\right| \leq \frac{L+2 c_{2}}{c_{1}}\right\}\right) \geq c_{1} \varepsilon,
$$

and the first inequality in (2.3) implies that

$$
\mu^{f}\left(\left\{z \in X^{f}:\left|c\left(t_{n}, z\right)-m_{n}\right| \leq \frac{L+2 c_{2}}{c_{1}}+2\right\}\right) \geq c_{1} \varepsilon
$$

for every $n \geq 1$. Since $\mu^{f}$ is mixing and $\lim _{n \rightarrow \infty} t_{n}=\infty$ by $(2.5), \lim _{n \rightarrow \infty} \nu_{t_{n}}$ $=\mu^{f} \times \mu^{f}$, where $\nu_{t_{n}}$ is the off-diagonal measure (1.5) with $\mu$ and $g$ replaced by $\mu^{f}$ and $t_{n}$.

We choose a countable dense subgroup $G \subset \mathbb{R}$ which contains the sequence $\left(t_{n}\right)$. Since $G$ is dense in $\mathbb{R}$ and $\mu^{f} \times \mu^{f}$ is ergodic under the diagonal $\mathbb{R}$-action $T^{f} \times T^{f}$ on $X^{f} \times X^{f}$, the measure $\mu^{f} \times \mu^{f}$ is also ergodic under the restriction of $T^{f} \times T^{f}$ to $G$, which implies that $\mu^{f}$ is weakly mixing under the restriction of $T^{f}$ to $G$. According to (2.6) and Proposition 1.3 there exist a homomorphism $\eta: G \rightarrow \mathbb{R}$ and a Borel map $b: X^{f} \rightarrow \mathbb{R}$ such that

$$
c(t, z)=\eta(t)+b\left(T_{t}^{f} z\right)-b(z)
$$


for every $t \in G$ and $\mu^{f}$-a.e. $z \in X^{f}$. From (2.4) we know that $\eta(t)=t$ for every $t \in G$, and the continuity of the map $t \mapsto c(t, \cdot)$ from $\mathbb{R}$ into $L^{1}\left(X^{f}, \mathcal{S}^{f}, \mu^{f}\right)$ in (2.2) guarantees that (2.7) holds for every $t \in \mathbb{R}$.

We re-trace our steps and conclude from (2.7) and (2.3) that the cocycle $\mathbf{f}^{\prime}: \mathbb{Z} \times X \rightarrow \mathbb{R}$, defined by

$$
\mathbf{f}^{\prime}(n, \cdot)=\mathbf{f}(n, \cdot)-n, \quad n \in \mathbb{Z},
$$

is bounded in $\mu$-measure. By [9, Theorem 11.8], [8, Theorem 5.2] or [10, Theorem 4.7] there exists a Borel map $b: X \rightarrow \mathbb{R}$ with $f(x)=1+b(T x)-b(x)$ for $\mu$-a.e. $x \in X$, which implies that $T^{f}$ is not even weakly mixing. This contradiction resulting from (2.5) proves the corollary.

\section{References}

[1] J. Aaronson and B. Weiss, Remarks on the tightness of cocycles, Colloq. Math. 84/85 (2000), 363-376.

[2] K. M. Khanin and Ya. G. Sinai, Mixing of some classes of special flows over a circle rotation, Funktsional. Anal. i Prilozhen. 26 (1992), no. 3, 1-21 (in Russian).

[3] A. V. Kochergin [A. V. Kočergin], On the absence of mixing in special flows over the rotation of the circle and in flows on a two-dimensional torus, Soviet Math. Dokl. 13 (1972), 949-952.

[4] -, Nonsingular saddle points and the absence of mixing, Math. Notes 19 (1976), $277-286$.

[5] D. G. Larman, Projecting and uniformising Borel sets with $K_{\sigma}$-sections $I$, Mathematika 19 (1972), 231-244.

[6] -, Projecting and uniformising Borel sets with $K_{\sigma}$-sections II, ibid. 20 (1973), 233-246.

[7] M. Lemańczyk, Sur l'absence de mélange pour des flots spéciaux au-dessus d'une rotation irrationnelle, Colloq. Math. 84/85 (2000), 29-41.

[8] C. C. Moore and K. Schmidt, Coboundaries and homomorphisms for nonsingular actions and a problem of H. Helson, Proc. London Math. Soc. 40 (1980), 443-475.

[9] K. Schmidt, Cocycles on Ergodic Transformation Groups, MacMillan (India), Delhi, 1977.

[10] - Amenability, Kazhdan's property T, strong ergodicity and invariant means for ergodic group actions, Ergodic Theory Dynam. Systems 1 (1981), 223-236.

[11] V. S. Varadarajan, Groups of automorphisms of Borel spaces, Trans. Amer. Math. Soc. 109 (1963), 191-220.

Mathematics Institute

University of Vienna

Strudlhofgasse 4

A-1090 Wien, Austria

E-mail: klaus.schmidt@univie.ac.at
Erwin Schrödinger Institute for Mathematical Physics Boltzmanngasse 9 A-1090 Vienna, Austria 